\title{
NeuroRegulation
}

\section{Connectivity-Guided EEG Biofeedback for Autism Spectrum Disorder: Evidence of Neurophysiological Changes}

\author{
Robert Coben ${ }^{1,4^{*}}$, Leslie Sherlin ${ }^{2}$, William J. Hudspeth ${ }^{3}$, Kevin McKeon ${ }^{1}$, Rachel \\ Ricca $^{4}$ \\ ${ }^{1}$ Neurorehabilitation \& Neuropsychological Services, New York, USA (Institution where the study was \\ conducted) \\ ${ }^{2}$ Southwest College of Naturopathic Medicine, Arizona; and Nova Tech EEG, Arizona, USA \\ ${ }^{3}$ Neuropsychometrix, California, USA \\ ${ }^{4}$ Integrated Neuroscience Services, Arkansas, USA
}

\begin{abstract}
*Address correspondence to: Robert Coben, Co-Director, Integrated Neuroscience Services, 86 West Sunbridge Drive Fayetteville, AR 72703, USA. Email: drcoben@integratedneuroscienceservices.com

Copyright: (C) 2014 Coben et al. This is an Open Access article distributed under the terms of the Creative Commons Attribution License (CC-BY).
\end{abstract}

\begin{abstract}
Recent studies have linked neural coherence deficits with impairments associated with Autism Spectrum Disorders (ASD). The current study tested the hypothesis that lowering neural hyperconnectivity would lead to decreases in autistic symptoms. Subjects underwent connectivity-guided EEG biofeedback, which has been previously found to enhance neuropsychological functioning and to lessen autistic symptoms. Significant reductions in neural coherence across frontotemporal regions and source localized power changes were evident in frontal, temporal, and limbic regions following this treatment. Concurrently, there were significant improvements on objective neuropsychological tests and parents reported positive gains (decreases in symptoms) following the treatment. These findings further validate EEG biofeedback as a therapeutic modality for autistic children and suggest that changes in coherence anomalies may be related to the mechanism of action.
\end{abstract}

Keywords: EEG biofeedback, autism, ASD, connectivity, coherence, LORETA

\section{Introduction}

The Centers for Disease Control and Prevention now indicates that the current prevalence of Autism Spectrum Disorder (ASD) is 1 in 68 (CDC, 2014). Furthermore, the U.S. Department of Education reported that from the 1992-1993 to 2001-2002 school years the rate of Autism increased 528\% and seems to continue to be on the rise (Safran, 2008; Yeargin-Allsopp, Rice, Karapurkar, Doernbert, Boyle, \& Murphy 2003). Ganz (2006) found that in the United States approximately $\$ 3.2$ million is spent to care for a single individual with Autism over the course of his or her lifetime, which in turn equates to a total cost of $\$ 35$ billion annually. 
Beyond the monetary costs, countless other collateral effects are felt by family members who care for those with ASD and by the individuals themselves.

Autism is a neurodevelopmental disorder characterized by a triad of impairment in social interaction, communication, and restricted patterns of behavior or interests (APA, 1994). The Diagnostic and Statistical Manual-Fourth Edition (DSM-IV) criteria states that autism cannot be diagnosed before three years of age; however, a recent survey has found that parents have reported autistic symptoms as early as 18 months and have even sought medical treatment before the age of two (APA, 1994; Filipek et al., 1999). The heterogeneous range of pervasive developmental disorders includes the following classifications: autistic disorder, Rett's syndrome, childhood disintegrative disorder, Asperger's disorder, and pervasive developmental disorder not otherwise specified (PDD-NOS; DSM-IV, APA, 1994). These classifications are categorized by a broad range of common symptoms coupled with levels of severity. Speech may be inflexible and unresponsive to the context as well as limited to echolalia or narrow topics of expertise in which discourse can proceed without conversational interplay (Belmonte, Beckel-Mitchener, Boulanger, Carper, \& Webb 2004). Moreover, social behaviors are often characterized by lack of interaction; play lacks cooperation in which the child usually confines himself or herself to playing on the periphery of the group. Additionally, the imagination of a child with ASD is usually deficient and the individual narrowly focuses on repetitive behaviors (Belmonte et al., 2004).

Recent research points to a theory of faulty neural connectivity as a mechanism underlying the symptoms of ASD (Baron-Cohen, 2004; Belmonte et al., 2004). "Connectivity" can be defined as any number of means of measuring the communication between two or more neural locations within the brain (Coben, 2007). Rippon, Brock, Brown, and Boucher (2007) suggested a model of Autism associated with information integration deficits resulting from reduced connectivity between specialized local neural networks and over-connectivity within individual neural assemblies, most notably within the frontal lobes. Over-connectivity, or hyperconnectivity, refers to excessive communication between neural locations in the brain. Over-connectivity of neural assemblies within and between the frontal lobes have been found to lead to disruptions in the integration of information from emotional, language, sensory, and automatic systems (Courchesne \& Pierce, 2005). Mizuno, Villalobos, Davies, Dahl, and Muller (2006) found though the use of fMRI data that individuals with ASD have areas of excessive connectivity within numerous neural locations, most notably the right postcentral and middle frontal regions as well as the left insula. Likewise, Buxhoeveden, Semendeferi, Schenker, and Courchesne (2004) reported evidence that Autism is a disorder of excessive connectivity within the frontal lobes, which in turn impacts the neural connectivity between the frontal cortex and other brain systems. Diffusion Tensor Imaging (DTI) studies of subjects with ASD have revealed evidence of the following neural abnormalities: increases in cerebral white matter volumes within the frontal cortex, abnormally small minicolumns in the frontal area, and abnormally long dendritic spines present in high densities. Variable resolution electromagnetic tomography (VARETA) images of patients with ASD also showed increased activity in the cerebellum, thalamus, hippocampus, parahippocampal, cuneus, cingulate, and lingual gyrus as well as in temporal, precentral, postcentral, parietal, and occipital cortical regions (Coben, Chabot, \& Hirschberg 2013). All of the aforementioned evidence is indicative of frontal dysfunction consistent with hyperconnectivity for subjects with ASD (Barnea-Goraly et al., 2004; Herbert et al., 2004; Buxhoeveden et al., 2004; Semendeferi et al., 2004; Belmonte et al., 2004). It has recently been hypothesized that reducing neural hyperconnectivity within the autistic brain can lead to improvements in realms such as, but not limited to, attention, self-regulatory functions, social behavior, and communication skills (Coben \& Myers, 2008). 
Electroencephalographic (EEG) analysis provides real time neural data of electrical activity across multiple regions with excellent temporal resolution. Cantor, Thatcher, Hrybyk, and Kaye (1986) conducted computerized EEG analyses of 11 children with ASD between the ages of 4 and 12 years, in contrast to three other groups of children: (a) 88 normal children, (b) a matched group of 18 mentally handicapped children, and (c) a group of 13 mental agematched normal toddlers. The findings indicated that children with ASD had significantly greater coherence between hemispheres in the beta band than mentally handicapped, normal children, or normal toddlers. Children in the autistic group had higher coherence in the alpha band than did those in the normal group, and had less interhemispheric and intrahemispheric asymmetry than participants in the normal or mentally handicapped group. "Coherence" is one means of measuring connectivity based on EEG data and represents a specific mathematical calculation of the cross-correlation between two waveforms within a frequency band. These findings would appear to suggest that the EEG is a useful and valid means of measuring coherence anomalies in this population. Continuing, Murias, Webb, Greenson, and Dawson (2007) used EEG analysis to assess connectivity in 18 adults with ASD in comparison to 18 healthy adult controls in eyes-closed resting states. Their results showed that there was locally elevated coherence in the ASD group, particularly within the left hemisphere in a low frequency (theta) band. In the lower alpha range $(8-10 \mathrm{~Hz})$, farreaching reduced coherence was evident for the ASD group within frontal regions, and between frontal regions and all other scalp locations (Murias et al., 2007). These results indicate a pattern of over-connectivity and under-connectivity in the brain of subjects with ASD. Coben, Clarke, Hudspeth, and Barry (2008) compared 20 children with and without ASD, matched for gender, age, and IQ. Findings were suggestive of dysfunctional integration of frontal and posterior sites with patterns of extensive coherence anomalies. Coben et al. (2013) studied 91 children with ASD and compared them to 91 normal controls. The findings showed differences for EEG power, asymmetry, and coherence. There was a combination of both hyper- and hypocoherence with high coherence over frontal regions and with low coherences across temporal and posterior brain regions.

Preliminary research suggests that EEG biofeedback may be an effective form of therapy for reducing autistic symptoms in children (Coben \& Padolsky, 2007; Jarusiewicz, 2002). EEG biofeedback enables the clinician to train the brain to work in a new, more efficient way through the use of underlying operant conditioning paradigms. This treatment involves providing a subject with visual and auditory "feedback" for particular neural behaviors (Monastra, Monastra, \& George, 2002). Through conditioning, the subject is taught to inhibit EEG frequencies that are excessively generated and to augment frequencies that are deficient. With continuous training and coaching, subjects are taught to maintain brainwave patterns concurrent with healthy neural functioning. Recently, Walker, Kozlowski, and Lawson (2007) presented evidence demonstrating the ability of neurofeedback training to successfully train neural functioning to more normal states, while simultaneously showing reductions in autistic symptoms. For more in-depth information regarding EEG biofeedback the interested reader is referred to Hammond (2007). The efficacy of EEG biofeedback for autistic children was initially assessed by Jarusiewicz (2002), in which she reported a $26 \%$ decrease in autistic symptoms in the experimental group and a 3\% reduction in a wait-list control group. Further, this therapy is a therapeutic intervention that can be achieved over the course of a few months, has no demonstrable side effects, and is useful for a wide array of disorders including ADHD, epilepsy, dyslexia, and other areas of functioning (Leins et al., 2007; Hammond, 2007; Lubar, Swartwood, Swartwood, \& O'Donnell, 2005; Egner \& Sterman, 2006; Evans \& Park, 1996). 
In our previous study (Coben \& Padolsky, 2007), we presented evidence supporting the efficacy of connectivity-guided EEG biofeedback for ASD. Parental judgment of clinical improvement was positive in $89 \%$ (33 of 37 subjects) of the experimental group. This was significantly different from the control group in which $83 \%$ of parents reported no change. Importantly, no subject in either group reported a worsening in autistic symptoms. Parental ratings on the Autism Treatment Evaluation Checklist (ATEC; Rimland \& Edelson, 2000) showed a $40 \%$ decrease in core autistic symptoms as a result of this intervention in the experimental group. Decreases in autistic symptoms were also found on the Gilliam Asperger's Disorder Scale (GADS; Gilliam, 2001), Gilliam Autism Rating Scale (GARS; Gilliam, 1995), Personality Inventory for Children, Second Edition (PIC-2; Lachar, \& Gruber, 2001), and Behavior Rating Inventory of Executive Function (BRIEF; Gioia, Isquith, Guy \& Kenworthy, 2000). In comparison, no such changes were evident in the wait-list control group. Finally, pre-post neuropsychological evaluations of attention, visual perceptual, language, and executive functioning revealed increases in the experimental group's neuropsychological performance, as much as a one standard deviation improvement per domain, by the completion of the study.

In the present study we expand on these previous findings by presenting data regarding changes in brain functioning. Source localized changes in EEG power and coherence will be explored. We hypothesize that connectivity-guided EEG biofeedback is an intervention capable of changing the autistic brain in a therapeutic manner. We further hypothesize that reducing neural hypercoherence underlies the above reviewed clinical efficacy of this approach.

\section{Method}

\section{Participants}

Thirty-seven children diagnosed with ASD were utilized for this study as the experimental group, while 12 children also diagnosed with ASD served as the wait-list control group. The groups did not differ significantly in terms of age, gender, race, handedness, other treatments, or severity of ASD as indicated by the ATEC. The experimental group received at least 20 sessions of connectivity-guided EEG biofeedback training, while the wait-list control group received no experimental treatment. Finally, the necessary informed consent was obtained and all procedures were fully explained to parents in order for their children to participate in the study.

The experimental group included 31 males and 6 females with ages ranging from 3.92 to 14.66 years with a mean age of 8.92 years. This group contained 36 Caucasians and one Asian American. Among the experimental group 56.8\% $(n=21)$ were diagnosed with PDDNOS, $18.9 \%(n=7)$ with Autism, $13.5 \%(n=5)$ with Asperger's syndrome, and $10.8 \%(n=4)$ with childhood disintegrative disorder. The wait-list control group included 10 males and two females with ages ranging from 5.83 to 10.92 years with an average age of 8.19 years.

\section{Materials}

EEG data collection. EEG data was collected in part as the basis for evaluating coherence differences in this study. EEG data was obtained under two conditions, eyes closed and eyes open. A stretchable electrode cap embedded with 19 sensors attached to the scalp was used to collect data, with frontal reference, prefrontal ground, and linked ears. Each recording lasted 20 minutes, where 10 minutes were spent in both conditions. All the data 
collected was manually artifacted and analyzed for measures of multivariate coherence/connectivity in NeuroRep (Hudspeth, 1999). Further, Neurometric Analysis System (NxLink, 2001; John, Prichep, Fridman, \& Easton, 1988) and Neuroguide (Thatcher, Walker, Biver, North, \& Curtin, 2003), both of which are FDA approved, were used to analyze absolute power, relative power, and coherence (Thatcher et al., 2003). The reliability and validity of quantitative EEG (QEEG) have been sufficiently assessed and confirmed (Thatcher et al., 2003).

QEEG involved recording and digitizing EEG readings based on the International 10/20 System of electrode placement utilizing the Deymed Diagnostic (2004) TruScan 32 Acquisition EEG System. This system included 32 channels with sampling at 128 cycles per second and filtering between $0.1-40 \mathrm{~Hz}$. All recordings were done with impedance less than $5 \mathrm{kOhms}$. The common mode rejection ratio for this system is $102 \mathrm{~dB}$ and the isolation mode rejection ratio is $140 \mathrm{~dB}$. QEEG analysis mathematically compares an individual EEG reading to matched normative samples for age and gender. Through this analysis inconsistencies in EEG neural functioning can be located and addressed. Moreover, it has been found that QEEG analysis provides reliable descriptors of normative brain activity (John et al., 1988). QEEG analyses were performed both before and after the administration of connectivity-guided EEG biofeedback.

Neurofeedback equipment. The NeuroCybernetics EEGer Training System (NeuroCybernetics Inc., 2006) was used to perform connectivity-guided EEG biofeedback training. The sensors (Grass Silver Disc 48" Electrodes with SafeLead protected terminals; Grass SafeLead, 2006) were applied to the subject's scalp to measure EEG activity. The signal was then fed back to the subject in visual and aural form based on relative amplitude/threshold values. The visual feedback consisted of simple graphics (presented in the form of computer games), providing a continuous display of the ratio of amplitude to threshold for each stream of data. The aural reward consisted of a pre-recorded sound file of a short quarter of a second beep, occurring no more often than once per every half second and activating when specific amplitude/coherence conditions were met (NeuroCybernetics Inc., 2006). Treatment was personalized to each individual on the basis of his or her original QEEG findings for power and coherence. Based on each participant's QEEG analysis, areas showing the most prominent hypercoherence were targeted for training. QEEG analysis involved analytically comparing a participant's individual EEG data to normative data indicative of such factors as age, gender, etc. For example, based on pretreatment QEEG analysis, one patient was found to have maximal hypercoherence in the right frontal region primarily in alpha. A protocol was designed for this patient to reward alpha (the frequency range of maximal hypercoherence) and to inhibit lower and higher frequency EEG activity at electrode sites F8/F7. This was achieved by increasing or rewarding the EEG amplitude between sequential EEG sensors on the scalp within the frequency range of maximal hypercoherence.

EEG amplitude can be defined as the difference between frequencies measured from an active and a reference sensor site. Therefore, increasing EEG amplitude (difference) implies decreasing coherence (similarity) between the EEG electrode sites; intrinsically this process causes two electrode sites to become more disparate. This is the crux of application of connectivity-EEG biofeedback as a means to decrease hypercoherence in children with ASD. Moreover, amplitude was chosen for training due to the relative ease in manipulation as described above. For a more in-depth discussion into the personalization of protocols, the interested reader is referred to Coben (2007). 
eLORETA. Exact Low Resolution Brain Electromagnetic Tomography (eLORETA) is a functional tomography that uses EEG data to create three-dimensional slices of neural activity highlighting areas of underactivity and overactivity (Pascual-Marqui, 1999). From these three-dimensional image slices of cortical grey matter, the neurofeedback clinician can better assess activity deep within the brain beyond the EEG detectable at the surface. To localize these power differences we chose eLORETA, which is the third incarnation of the LORETA system. eLORETA is currently considered the most exact version, and has been evaluated and found to be a useful tool for localizing power differences (Pascual-Marqui, 2007). Further, the empirical validity of eLORETA has been sufficiently substantiated (Pascual-Marqui, Esslen, Kochi, \& Lehmann, 2002).

Assessment scales. GARS is a behavioral checklist. This scale is comprised of four subtests (Stereotyped Behaviors, Communication, Social Interaction, and Developmental Disturbances) of 14 items each. The scale was normed on a sample of 1,092 children and young adults (aged 2 to 28) across 46 U.S. states, the District of Columbia, Puerto Rico, and Canada. The internal consistency reliability coefficients for all subtests and total Autism Quotient range from .88 to .96. The stability or test-retest reliability ranges from .81 to .88 for all subtests and total Autism Quotient. These results indicate high levels of stability required for pre-post treatment assessment of individuals with ASD. The construct validity was confirmed by analyses finding that: Items of the subscales are representative of the behaviors associated with Autism; GARS scores strongly relate to each other and to performance on other screening tests for Autism; GARS scores are not related to age; and individuals with other diagnoses score differentially on the GARS. The GARS is a scale shown to discriminate between autistic and non-autistic subjects with a $90 \%$ accuracy rate.

GADS is a behavioral rating scale. The GADS consists of 32 items divided into four subscales including: Social Interaction (10 items), Restricted Patterns of Behavior (8 items), Cognitive Patterns ( 7 items), and Pragmatic Skills (7 items). The GADS was normed on a sample of 371 individuals (aged 3 to 22; males [ $n=314]$, females [ $n=57]$ ) diagnosed with Asperger's disorder from across 46 U.S. states, the District of Columbia, Canada, Great Britain, Mexico, Australia, and other countries. Internal consistency reliability coefficients ranged from .87 to .95 for total Asperger's Disorder Quotient across samples of children with and without identified disabilities. The test-retest reliability for the Asperger's Disorder Quotient is .93 $(p<.01)$. These results indicate that the GADS has a high level of stability for use as a pre-post treatment measure of individuals with Asperger's disorder. Construct validity was indicated by analyses finding that: GADS scores are minimally related to age; items on the subscales are representative of behaviors associated with Asperger's disorder; persons with other diagnoses score differentially; GADS scores are strongly related to each other and performance on other tests that screen for serious behavioral disorders; and the GADS can discriminate among individuals with Asperger's disorder and those with behavioral disorders. The GADS has been found to have an $83 \%$ accuracy rate in discriminating Asperger's and non-Asperger's subjects (Gilliam, 2001).

\section{Procedure.}

A diagnostic interview was conducted with the parents to ascertain core behavioral, cognitive, and social/emotional issues of concern as part of a comprehensive neurodevelopmental history. Additionally, all participants involved in this study met the criteria for either: autistic disorder, Asperger's disorder, childhood disintegrative disorder, or PDD-NOS as described by the DSM-IV (APA, 1994). A second inclusion criteria for this study were scores on the GADS and the GARS. Only subjects with a total Asperger's 
Disorder Quotient of 70 or greater on the GADS or an Autism Quotient of 70 or greater on the GARS were used in this study.

All participants underwent QEEG analysis both before the start of connectivity-guided EEG biofeedback training as well as at the completion of no less than 20 sessions. A QEEG was performed before the administration of connectivity-guided EEG biofeedback in order to assess baseline levels of hyperconnectivity, coherence, and power. Again, this baseline is assessed by analytically comparing each participant's individual EEG data to a normative QEEG prior to treatment. Based on this original analysis, personalized EEG biofeedback protocols were designed so as to optimally and efficiently decrease hypercoherence in each subject. QEEG analysis performed after the administration of connectivity-guided biofeedback was used in tandem with pre-condition analysis to assess significant changes.

\section{Data Analysis.}

Source-localized (eLORETA) measures for absolute power were used as the initial set of dependent variables. In regards to EEG data collection, average cross-spectral matrices were computed for bands delta $(2-3.5 \mathrm{~Hz})$, theta $(4-7.5 \mathrm{~Hz})$, alpha $(8-12.5 \mathrm{~Hz})$, beta $(13-21$ $\mathrm{Hz}$ ), low frequency $(2-7 \mathrm{~Hz})$, high frequency $(13-32 \mathrm{~Hz})$, alpha1 $(8-10 \mathrm{~Hz})$, alpha2 (10-12 $\mathrm{Hz})$, beta1 $(12-16 \mathrm{~Hz})$, beta2 $(16-20 \mathrm{~Hz})$, beta3 $(20-24 \mathrm{~Hz})$, beta4 $(24-28 \mathrm{~Hz})$, and beta5 (28-32 Hz).

For every frequency band and subject in the two groups, the current density modules at each voxel (current density amplitude) were smoothed with a three-dimensional moving average filter, normalized, and finally log-transformed. Log-transformation of power estimates is routinely performed in EEG and eLORETA to approximate data Gaussianity (John, Prichep, \& Easton, 1987). With eLORETA, some smoothing is advisable to reduce anatomical and localization errors due to inter-individual differences in head geometry and electrodes placement. In general, local maxima can be visualized in slightly different locations. Spatial normalization consists of normalizing the square root of the sum of squared current density values for each subject at all voxels to equal unity. This manipulation eliminates confounding variables such as the inter-individual variability in skull thickness and electrode impedance, without constraining the analysis on relative power measures. Current density amplitude estimates computed and preprocessed as described provided the data for statistical analysis.

To compare the current density amplitude of the two conditions, we used the randomizationpermutation multiple comparison t-max approach (Congedo, Finos, \& Turkheimer, 2004) that has recently been utilized by Sherlin et al. (2007). Data-permutation approaches can adaptively account for the correlation structure of the variables, an embedded feature of all electrophysiological measurements (Holmes, Blair, Watson, \& Ford, 1996). We performed one test for each of the 13 frequency band-pass regions (delta, theta, alpha, beta, low frequency, high frequency, alpha1, alpha2, beta1, beta2, beta3, beta4, and beta5). For the whole data set (2,394 $\times 9$ variables), voxel-by-voxel within $t$-tests were computed; this is the $t$-test for paired designs. The mean of the pre-condition $(A)$ is compared to the mean of the post-condition (B). Individuals in the two conditions are the same. The test-statistic is the well-known student-t, with positive values indicating mean $(A)>$ mean $(B)$, and negative values indicating mean $(A)<$ mean $(B)$. In this test the mean of two conditions are compared. A threshold of significance (if the global null hypothesis was false) was then computed by the t-max method. For all bands, we tested the hypothesis that the mean 
LORETA current density amplitude of the two conditions differed by subtracting the values for the pre-condition from the post-condition (Congedo et al., 2004).

The eLORETA variables for each subject in each group (pre-post training) cross-spectral matrices were computed and averaged over 4-second epochs resulting in one cross-spectral matrix for each subject and for each of the discrete frequencies within each band. Based on previous LORETA analyses (Lubar, Congedo, \& Askew, 2003), we used a rectangular window. Sliding overlapping windows (overlap 93.8\%) allowed reliable and smooth spectral estimates. The LORETA-Key software package (Pascual-Marqui, Michel, \& Lehmann, 1994) was used to compute LORETA current density in the frequency domain directly from the average cross-spectral matrix (Frei et al., 2001). This LORETA implementation incorporates a 3-shell spherical head model registered to recognized anatomical brain atlas (Talairach \& Tournoux, 1988), and makes use of EEG electrode coordinates derived from crossregistration between spherical and realistic head geometry (Towle et al., 1993). The solution space is restricted to cortical gray matter using the digitized probability atlas of the Brain Imaging Center at the Montreal Neurological Institute (Collins, Neelin, Peters, \& Evans, 1994), divided in 2,394 voxels measuring $7 \times 7 \times 7 \mathrm{~mm}$ ).

To analyze coherence, subject groups were first prepared in the NeuroRep program NDAC. NDAC allows the user to identify and select a subset to compile raw connectivity indices for 171 pairwise combinations of 19 electrodes, with each having five frequency bands: delta $(0.5-3.5 \mathrm{~Hz})$, theta $(3.5-7 \mathrm{~Hz})$, alpha $(7-13 \mathrm{~Hz})$, beta $(13-22 \mathrm{~Hz})$ and total (amplifier bandwidth) and then compute 171 final group means, standard deviations, skewness, and kurtosis. Once the groups were prepared, the statistical program Compare was used to test for significant changes in coherence. Compare allows the user to compute correlated Student's $t$-tests between average connectivity indices for 171 pairwise electrode combinations (from NDAC). The significance of the $t$-test probabilities was evaluated with False Discovery Rate methods (Benjamini \& Hochberg, 1995; Miller et al., 2001) to control for multiple comparison errors. All connectivity indices can optionally be Fischer ztransformed to improve Gaussianity of the resulting distribution. The total band limited refers to an EEG amplifier bandwidth of $0.5-40 \mathrm{~Hz}$. Recursive filter bandwidths refer to four exacted bands: delta $(0.5-3.5 \mathrm{~Hz})$, theta $(3.5-7 \mathrm{~Hz})$, alpha $(7-13 \mathrm{~Hz})$, beta $(13-22 \mathrm{~Hz})$. The program compares means by use of Pearson correlations and correlated $t$-tests.

Additionally, normalization of connectivity values can be achieved by using Fisher's z-score transformation. Further, Compare accounts for multiple comparison error by use False Discovery Rate (FDR; Benjamini \& Hochberg, 1995) threshold values for judging significance. Connectivity indices were computed with software derived from the "COHER" programs written by Michael Hrybyk (Thatcher, Krause \& Hrybyk, 1986) and were widely used in commercial computerized EEG software: Quantitative Signal Imaging, NeuroRep, NeuroData, Lexior, and NeuroGuide. The routines implement the equation reported in Bendat and Piersol (1980) and they include the results from a calibration EEG recording. To the authors' knowledge, all commercial programs produce results identical to COHER's results for the EEG calibration file. In this equation, the signals are normalized over the entire record to minimize the influences of signal amplitudes and thereby emphasize the relationship between the pair of EEG profiles (Bendat \& Piersol, 1980). The values produced by Compare (coherence analyses) were a second set of dependant variables for this study.

\section{Results}

There were significant differences between pre- and post-conditions for source-localized absolute power. The maximum t-statistic, or maximum t-value across the entire volume, for 
each frequency band follows. Absolute power significant t-values: delta (-2.63), theta $(-2.43)$, and low frequency $(-2.57)$.

Figures 1 through 4 illustrate the statistically significant differences between the two conditions in absolute power along with the Brodmann's Area (Brodmann, 1909/2005) label of the voxel with maximum differences. eLORETA current source density is displayed in the given frequency band. Coordinates and t-values for the maximal different voxel are printed above the picture of the sagittal section. All t-statistics that are positive are displayed in red (the mean of post-condition is greater than the mean of the pre-condition). All t-statistics that are negative are displayed in blue (the mean of the post-condition is less than the mean of the pre-condition). Displayed are the horizontal (left), sagittal (middle), and coronal (right) sections through the voxel with maximal t-statistic. Further, errors associated with multiple comparisons were accounted for by our implementation of the randomization-permutation multiple comparison t-max approach (Congedo et al., 2004). Only significant results (images) are shown (Refer to Figures 1 through 4).

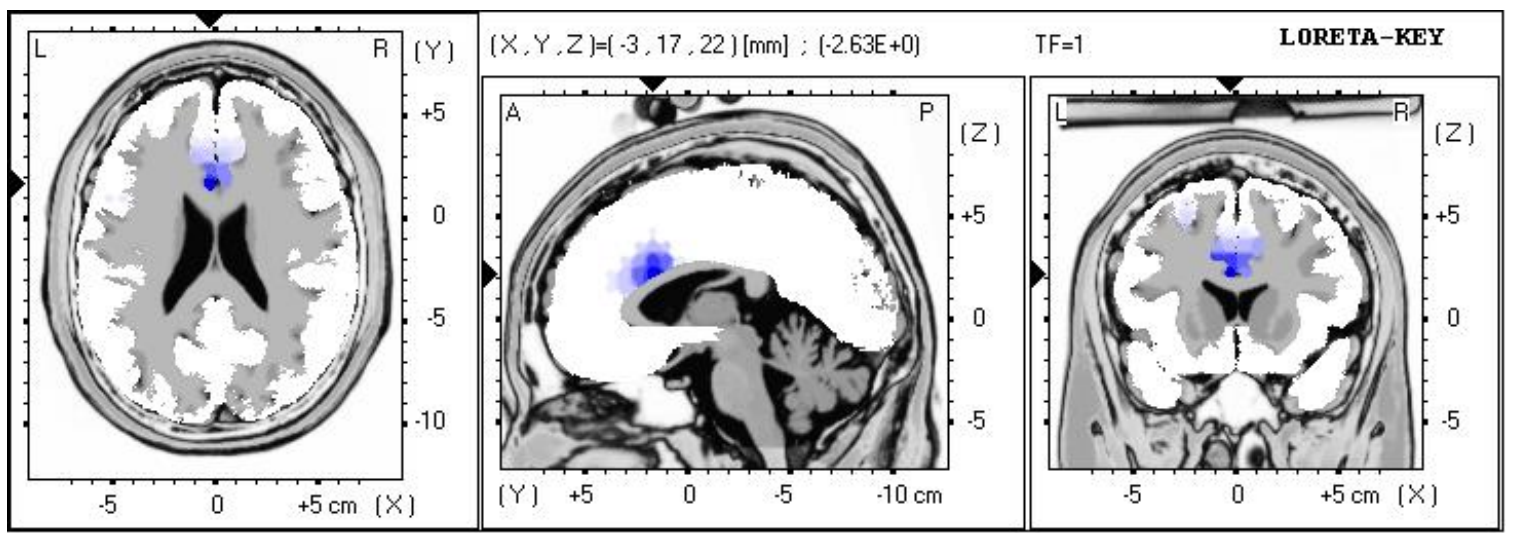

Figure 1. eLORETA analysis showing source localization of absolute delta power. Significant differences localized to Brodmann area 33, anterior cingulate, and limbic lobe.

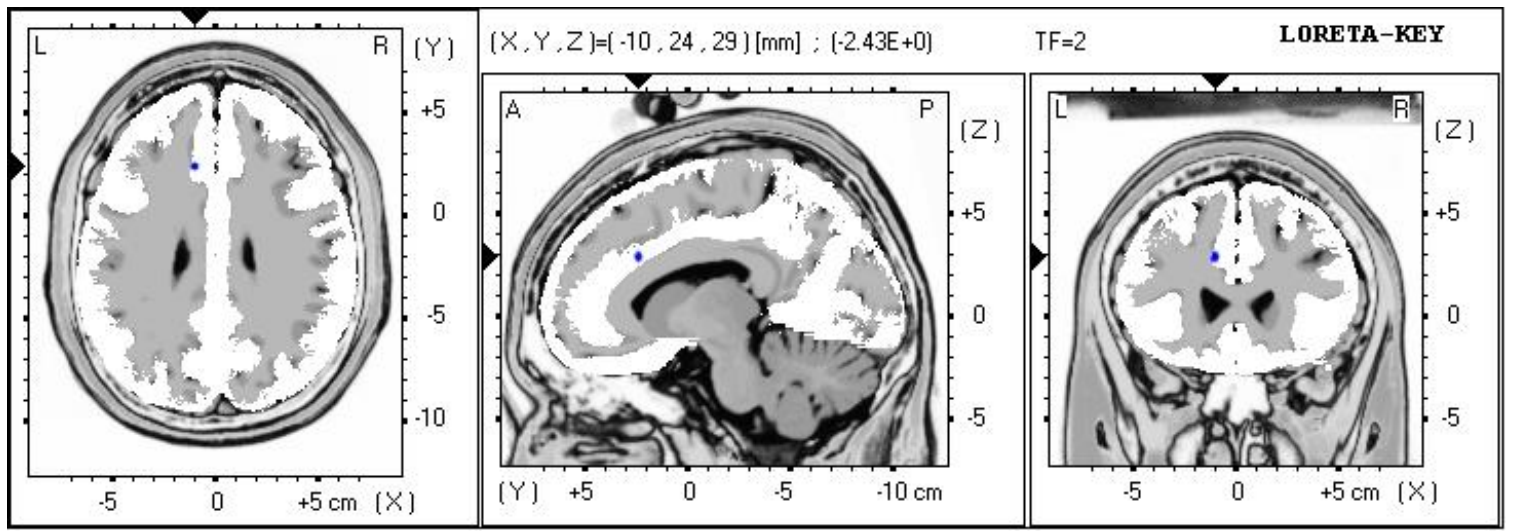

Figure 2. eLORETA analysis showing source localization of absolute theta power. Significant differences localized to Brodmann area 32, anterior cingulate, and limbic lobe. 


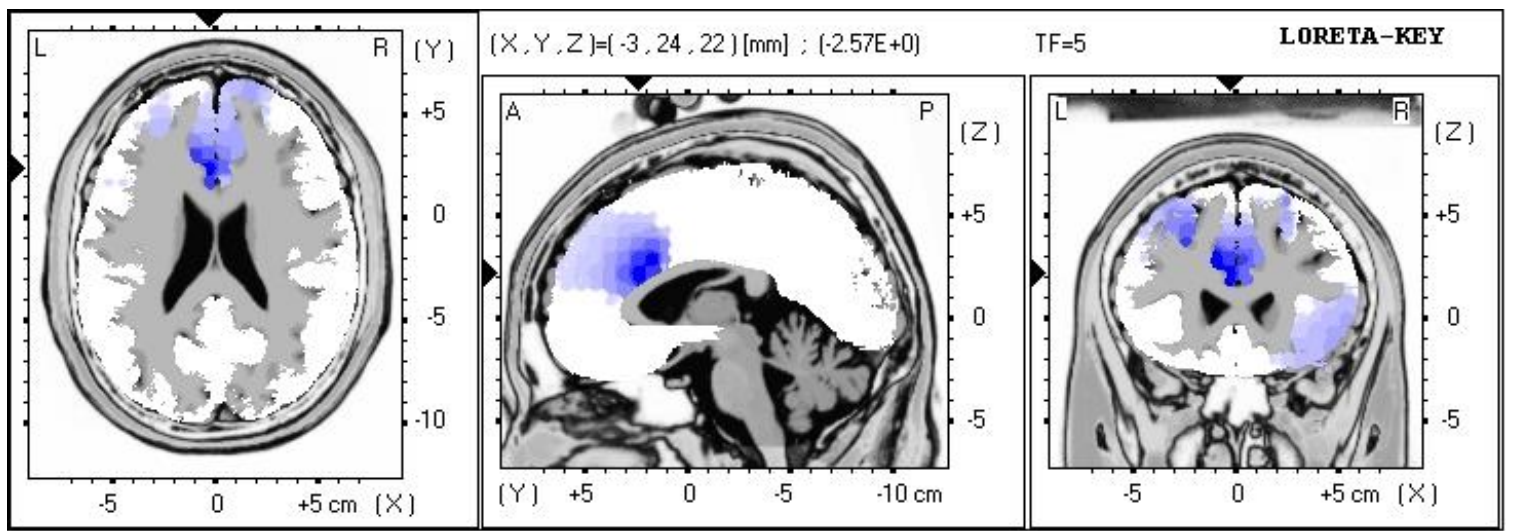

Figure 3. eLORETA analysis showing source localization of absolute low frequency power. Significant differences localized to Brodmann area 24, anterior cingulate, and limbic lobe.

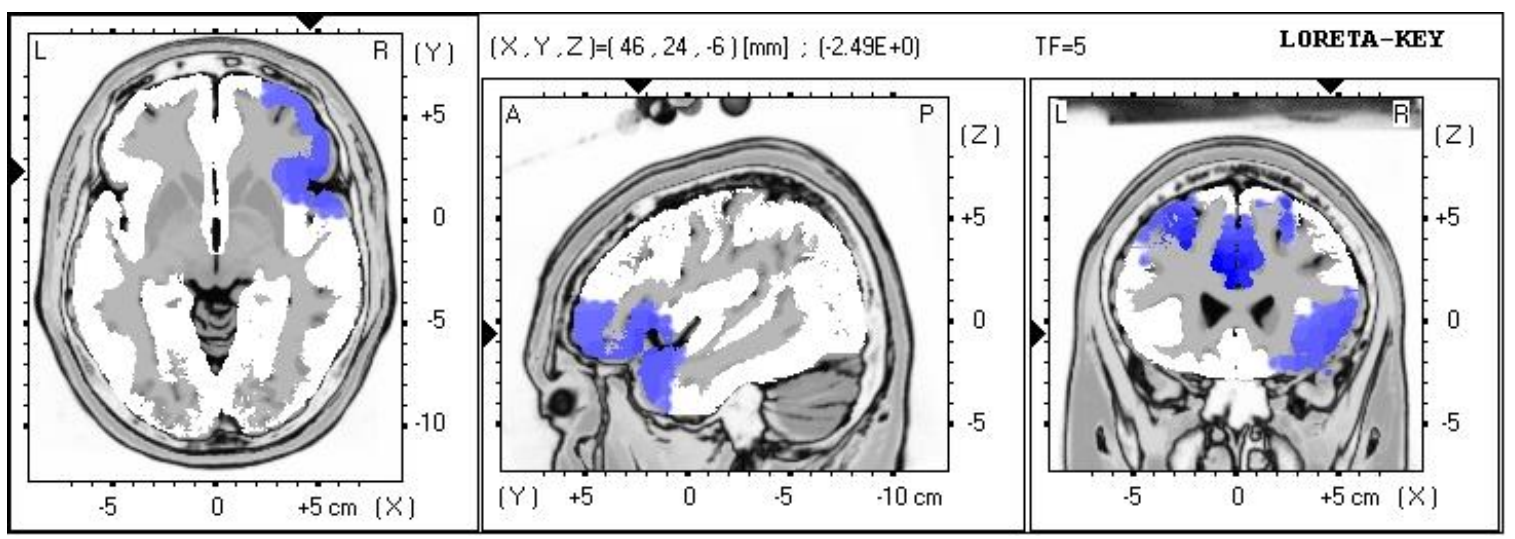

Figure 4. eLORETA analysis showing source localization of absolute low frequency power. Significant differences localized to Brodmann areas 10 and 47, frontal gyrus, and frontal lobe.

The significant differences between the pre- and post-conditions were localized to decreased slow wave activity $(2-7 \mathrm{~Hz})$ in the anterior cingulate and the right frontal gyrus. The strongest findings of decreased slow activity were found in Brodmann areas 32 and 33 that are located in the associational cortical area of the frontal lobes and that participate in prefrontal cortical networks, which are thought to govern personal and social behavior, emotion, and decision-making (Salloway, Malloy, \& Duffy, 2001; Courchesne \& Pierce, 2005). Brodmann area 24 is located in associational cortical area in the anterior part of the cingulate gyrus. This area is a cortical component of the limbic system that is involved in emotional processing, the control of facial expressions, and the affective dimensions of pain (Williams, White, \& Mace, 2005). Additionally, there was decreased low frequency band absolute power in Brodmann areas 10 and 47 of the right hemisphere. This area is in the associational cortical area in the anterior-polar prefrontal region of the frontal lobes and participates in prefrontal cortical networks that govern executive functions (Koechlin \& Hyafil, 2007). 
Pre- and post-EEG data groups were prepared and generated in NDAC. Differences between these groups, in terms of coherence values, were analyzed in the statistical package Compare. This statistical package made comparisons between 171 pairwise means using Pearson correlations and correlated $t$-tests. Two-tailed probabilities were utilized for paired $t$-tests in the delta, theta, alpha, beta bands, and across the total frequency as well. FDR indices were used to control for errors due to multiple comparisons (Benjamini \& Hochberg, 1995). Similar to eLORETA, pre-condition scores were subtracted from postcondition scores in Compare. Blue results indicated a decrease in connectivity, while red results indicated increases in connectivity. As figures 5 through 7 show, the statistical analyses indicated numerous significant reductions in connectivity between neural locations predominately in the alpha and beta bands, as well as in the total coherence.

Figure 5 shows the statistical analysis of the alpha band values. Eleven electrode pairs were found to have significant decreases in neural connectivity within this band, while only one pair was found to have a significant increase in connectivity.

\begin{tabular}{|c|c|c|c|c|c|c|c|c|c|c|c|c|c|c|c|c|c|c|}
\hline$F p 2$ & -0.562 & & & & & & & & & & & & & & & & & \\
\hline$F 7$ & \begin{tabular}{|l|}
-0.814 \\
\end{tabular} & $-0.511 \mid$ & & & & & & & & & & & & & & & & \\
\hline$F 8$ & -0.133 & -0.089 & -0.400 & & & & & & & & & & & & & & & \\
\hline$F 3$ & 0.154 & 0.440 & -0.042 & -0.298 & & & & & & & & & & & & & & \\
\hline$F 4$ & -0.272 & -0.991 & -0.028 & 0.874 & -0.051 & & & & & & & & & & & & & \\
\hline$T 3$ & -0.090 & -0.653 & -0.071 & \begin{tabular}{|l|}
-0.807 \\
\end{tabular} & \begin{tabular}{|c|}
-0.039 \\
\end{tabular} & -0.130 & & & & & & & & & & & & \\
\hline$T 4$ & -0.000 & -0.001 & -0.134 & -0.003 & -0.001 & -0.001 & 0.342 & & & & & & & & & & & \\
\hline $\mathrm{C} 3$ & -0.058 & -0.069 & -0.025 & -0.019 & -0.038 & -0.010 & -0.123 & -0.012 & & & & & & & & & & \\
\hline$C 4$ & -0.002 & -0.008 & -0.002 & -0.017 & -0.005 & -0.001 & -0.288 & -0.163 & -0.023 & & & & & & & & & \\
\hline 75 & 0.558 & 0.159 & -0.245 & 0.158 & -0.246 & 0.424 & -0.220 & 0.104 & -0.202 & 0.831 & & & & & & & & \\
\hline$T 6$ & 0.011 & 0.103 & 0.003 & 0.806 & 0.017 & -0.859 & 0.041 & -0.268 & \begin{tabular}{|l}
0.433 \\
\end{tabular} & -0.487 & 0.179 & & & & & & & \\
\hline P3 & -0.191 & -0.916 & -0.043 & -0.827 & \begin{tabular}{|l|}
-0.107 \\
\end{tabular} & \begin{tabular}{|l|}
-0.076 \\
\end{tabular} & -0.104 & -0.745 & -0.162 & -0.128 & 0.910 & 0.395 & & & & & & \\
\hline$P 4$ & -0.743 & -0.914 & -0.926 & -0.705 & $-0.341 \mid$ & -0.141 & 0.878 & -0.332 & -0.118 & -0.625 & 0.589 & 0.563 & -0.519 & & & & & \\
\hline 01 & 0.560 & \begin{tabular}{|l|}
0.133 \\
\end{tabular} & \begin{tabular}{|l|}
-0.882 \\
\end{tabular} & 0.190 & -0.437 & 0.874 & -0.504 & 0.262 & -0.685 & 0.870 & 0.782 & 0.234 & 0.942 & \begin{tabular}{|l|l|} 
\\
\end{tabular} & & & & \\
\hline 02 & 0.101 & 0.007 & 0.092 & 0.081 & 0.210 & 0.189 & 0.612 & 0.689 & -0.931 & 0.643 & 0.774 & 0.084 & -0.382 & \begin{tabular}{|l|} 
\\
\end{tabular} & \begin{tabular}{|l|}
-0.228 \\
\end{tabular} & & & \\
\hline $\mathrm{Fz}$ & 0.608 & 0.307 & -0.031 & -0.264 & -0.111 & \begin{tabular}{|l|}
-0.072 \\
\end{tabular} & -0.042 & -0.002 & -0.015 & $-0,003$ & -0.960 & 0.261 & -0.052 & \begin{tabular}{|l|}
-0.194 \\
\end{tabular} & \begin{tabular}{|l}
-0.835 \\
\end{tabular} & 0.379 & & \\
\hline $\mathrm{Cz}$ & -0.193 & -0.255 & -0.026 & $-0,068$ & -0.071 & -0.017 & -0.148 & -0.006 & -0.040 & -0.023 & -0.431 & -0.852 & -0.060 & -0.133 & -0.932 & -0.787 & -0.039 & \\
\hline$P_{z}$ & -0.016 & -0.108 & -0.017 & -0.013 & -0.048 & -0.009 & -0.380 & -0.058 & -0.185 & -0.143 & -0.886 & -0.654 & -0.629 & -0.441 & -0.581 & -0.635 & -0.016 & -0.104 \\
\hline & $F_{p y t}$ & $\mathrm{Fp} 2$ & $F 7$ & $F 8$ & $F 3$ & F4 & $T 3$ & $T 4$ & $\mathrm{C} 3$ & C4 & $T 5$ & 76 & $P 3$ & P4 & of & 02 & $F_{z}$ & $\mathrm{Cz}$ \\
\hline
\end{tabular}

Figure 5. Compare findings of changes in alpha coherence, with a False Discovery Rate (FDR) of alpha $p<=.0053$.

As Figure 6 shows, the analyses of the beta band found 43 electrode pairs to have significant decreases in neural connectivity. 


\begin{tabular}{|c|c|c|c|c|c|c|c|c|c|c|c|c|c|c|c|c|c|c|}
\hline $\mathrm{Fp} 2$ & -0.023 & & & & & & & & & & & & & & & & & \\
\hline$F 7$ & -0.151 & -0.035 & & & & & & & & & & & & & & & & \\
\hline$F 8$ & -0.007 & -0.014 & -0.011 & & & & & & & & & & & & & & & \\
\hline$F 3$ & -0.604 & -0.247 & -0.198 & -0.024 & & & & & & & & & & & & & & \\
\hline$F 4$ & -0.019 & -0.121 & -0.016 & -0.391 & -0.045 & & & & & & & & & & & & & \\
\hline 73 & -0.059 & -0.337 & -0.177 & -0.485 & -0.056 & -0.054 & & & & & & & & & & & & \\
\hline$T 4$ & -0.001 & -0.030 & -0.086 & -0.183 & -0.007 & -0.090 & 0.526 & & & & & & & & & & & \\
\hline $\mathrm{C} 3$ & -0.051 & -0.010 & -0.055 & -0.014 & -0.038 & $-0,009$ & -0.079 & -0.002 & & & & & & & & & & \\
\hline CA & -0.003 & -0.003 & -0.002 & -0.030 & -0.005 & -0.012 & -0.026 & -0.266 & -0.004 & & & & & & & & & \\
\hline$T 5$ & -0.650 & 0.270 & -0.056 & 0.147 & -0.028 & -0.499 & -0.227 & 0.152 & -0.056 & -0.536 & & & & & & & & \\
\hline 76 & 0.438 & $\mid-0.176$ & 0.274 & -0.197 & -0.534 & .0 .042 & 0.070 & -0.262 & -0.267 & -0.159 & -0.624 & & & & & & & \\
\hline P3 & -0.005 & -0.004 & -0.010 & -0.007 & -0.003 & -0.002 & -0.069 & -0.076 & -0.004 & -0.020 & -0.758 & -0.484 & & & & & & \\
\hline$P 4$ & -0.046 & -0.007 & -0.032 & -0.037 & -0.006 & -0.018 & -0.134 & -0.174 & -0.008 & \begin{tabular}{|c|}
-0.188 \\
\end{tabular} & -0.448 & -0.581 & -0.100 & & & & & \\
\hline O1 & 0.529 & 0.234 & -0.312 & 0.508 & -0.018 & -0.076 & -0.260 & 0.939 & $|-0.064|$ & -0.209 & 0.538 & -0.689 & $-0.917 \mid$ & -0.245 & & & & \\
\hline $\mathrm{O} 2$ & 0.420 & 0.791 & 0.159 & -0.481 & -0.250 & -0.115 & -0.298 & -0.714 & -0.040 & 0.990 & -0.328 & 0.142 & -0.755 & 0.196 & -0.077 & & & \\
\hline $\mathrm{Fz}$ & -0.094 & \begin{tabular}{|l}
-0.310 \\
\end{tabular} & -0.012 & -0.051 & -0.040 & \begin{tabular}{|c|}
-0.139 \\
\end{tabular} & -0.023 & -0.027 & -0.013 & -0.006 & -0.041 & -0.175 & -0.003 & -0.008 & -0.032 & -0.177 & & \\
\hline $\mathrm{Cz}$ & -0.022 & \begin{tabular}{|l|}
-0.019 \\
\end{tabular} & -0.006 & -0.039 & -0.007 & -0.068 & -0.033 & $-0,073$ & -0.005 & -0.022 & -0.136 & -0.179 & -0.012 & \begin{tabular}{|l|}
-0.040 \\
\end{tabular} & -0.110 & -0.507 & -0.017 & \\
\hline$P z$ & -0.002 & -0.001 & -0.005 & -0.006 & -0.002 & -0.008 & -0.036 & -0.048 & -0.004 & -0.063 & -0.598 & -0.698 & -0.214 & -0.325 & -0.408 & 0.849 & -0.005 & -0.023 \\
\hline & Fpt & $F_{p 2}$ & $F 7$ & $F 8$ & $F 3$ & $F 4$ & $T 3$ & $T 4$ & $\mathrm{C} 3$ & $\mathrm{C} 4$ & T5 & 76 & $P 3$ & $P 4$ & of & $\mathrm{O} 2$ & $F_{Z}$ & $\mathrm{Cz}$ \\
\hline
\end{tabular}

Figure 6. Compare findings of changes in beta coherence, with a False Discovery Rate (FDR) of alpha $p<=.064$.

As shown in Figure 7, the analyses for the total connectivity revealed 42 neural locations to have significant reductions in connectivity. The total connectivity is an analysis of connectivity encompassing the entire EEG spectrum $(0.5-22 \mathrm{~Hz})$.

\begin{tabular}{|c|c|c|c|c|c|c|c|c|c|c|c|c|c|c|c|c|c|c|}
\hline$F_{p 2}$ & -0.059 & & & & & & & & & & & & & & & & & \\
\hline$F 7$ & -0.432 & -0.339 & & & & & & & & & & & & & & & & \\
\hline$F 8$ & -0.016 & -0.016 & -0.243 & & & & & & & & & & & & & & & \\
\hline$F 3$ & 0.832 & -0.754 & -0.135 & -0.357 & & & & & & & & & & & & & & \\
\hline$F 4$ & -0.005 & -0.081 & -0.019 & -0.789 & -0.016 & & & & & & & & & & & & & \\
\hline 73 & -0.047 & -0.089 & -0.095 & -0.032 & -0.045 & -0.022 & & & & & & & & & & & & \\
\hline$T 4$ & -0.002 & -0.040 & -0.007 & -0.342 & -0.001 & -0.025 & -0.055 & & & & & & & & & & & \\
\hline $\mathrm{C} 3$ & -0.016 & -0.048 & -0.083 & -0.225 & -0.045 & -0.039 & -0.091 & -0.014 & & & & & & & & & & \\
\hline$C 4$ & -0.000 & -0.015 & -0.008 & -0.441 & -0.005 & -0.015 & -0.022 & -0.100 & -0.024 & & & & & & & & & \\
\hline 75 & -0.004 & -0.010 & -0.040 & -0.008 & -0.024 & -0.017 & -0.083 & -0.060 & -0.126 & -0.103 & & & & & & & & \\
\hline 76 & -0.001 & -0.010 & -0.003 & -0.068 & -0.003 & -0.013 & -0.014 & \begin{tabular}{|l|}
-0.139 \\
\end{tabular} & -0.032 & -0.183 & -0.228 & & & & & & & \\
\hline P3 & -0.002 & -0.006 & -0.022 & -0.037 & -0.020 & \begin{tabular}{|r|}
-0.023 \\
\end{tabular} & -0.082 & -0.090 & -0.195 & -0.164 & -0.617 & -0.335 & & & & & & \\
\hline P4 & -0.003 & -0.012 & -0.009 & -0.137 & -0.011 & -0.036 & -0.012 & -0.130 & -0.034 & -0.432 & -0.087 & -0.296 & -0.095 & & & & & \\
\hline O1 & -0.008 & -0.008 & -0.027 & -0.012 & -0.065 & \begin{tabular}{|l|}
-0.067 \\
\end{tabular} & -0.126 & -0.153 & -0.286 & -0.377 & 0.238 & -0.910 & -0.943 & -0.229 & & & & \\
\hline 02 & -0.008 & -0.011 & -0.014 & -0.052 & \begin{tabular}{|r|}
-0.030 \\
\end{tabular} & \begin{tabular}{|l|}
-0.106 \\
\end{tabular} & -0.020 & -0.425 & -0.122 & -0.928 & -0.079 & 0.096 & -0.086 & 0.195 & \begin{tabular}{|l|}
-0.067 \\
\end{tabular} & & & \\
\hline$F z$ & -0.031 & -0.440 & -0.028 & -0.222 & -0.161 & -0.040 & -0.029 & -0.006 & -0.033 & $-0,010$ & -0.024 & -0.010 & $-0.020 \mid$ & $|-0.026|$ & $\mid-0.095$ & $|-0.086|$ & & \\
\hline $\mathrm{Cz}$ & -0.020 & -0.093 & -0.046 & -0.557 & -0.045 & -0.107 & -0.040 & -0.069 & -0.077 & -0.154 & -0.111 & -0.100 & -0.149 & -0.192 & -0.369 & -0.329 & -0.068 & \\
\hline$P z$ & -0.002 & -0.008 & -0.013 & -0.102 & -0.018 & -0.034 & -0.050 & -0.073 & -0.123 & -0.263 & -0.430 & -0.421 & -0.355 & -0.430 & -0.493 & -0.531 & -0.028 & -0.261 \\
\hline & Fof & Fo2 & $F 7$ & F8 & $F 3$ & $F 4$ & $T 3$ & $T 4$ & $\mathrm{C} 3$ & C4 & $T 5$ & $T 6$ & P3 & P4 & Of & $\mathrm{O} 2$ & $F z$ & $\mathrm{Cz}$ \\
\hline
\end{tabular}

Figure 7. Compare findings of changes in total coherence, with a False Discovery Rate (FDR) of alpha $p<=.0164$. 
Figure 8 presents a graphical representation of our Compare calculations. Blue lines between focal points indicated deceases in connectivity, while orange lines indicated increases in connectivity. Moreover, only significant findings are shown. As our analysis shows, connectivity-guided EEG biofeedback predominately produced reductions in neural connectivity. Further, these decreases in connectivity seemed to occur mostly within the frontotemporal region, especially on the right side.

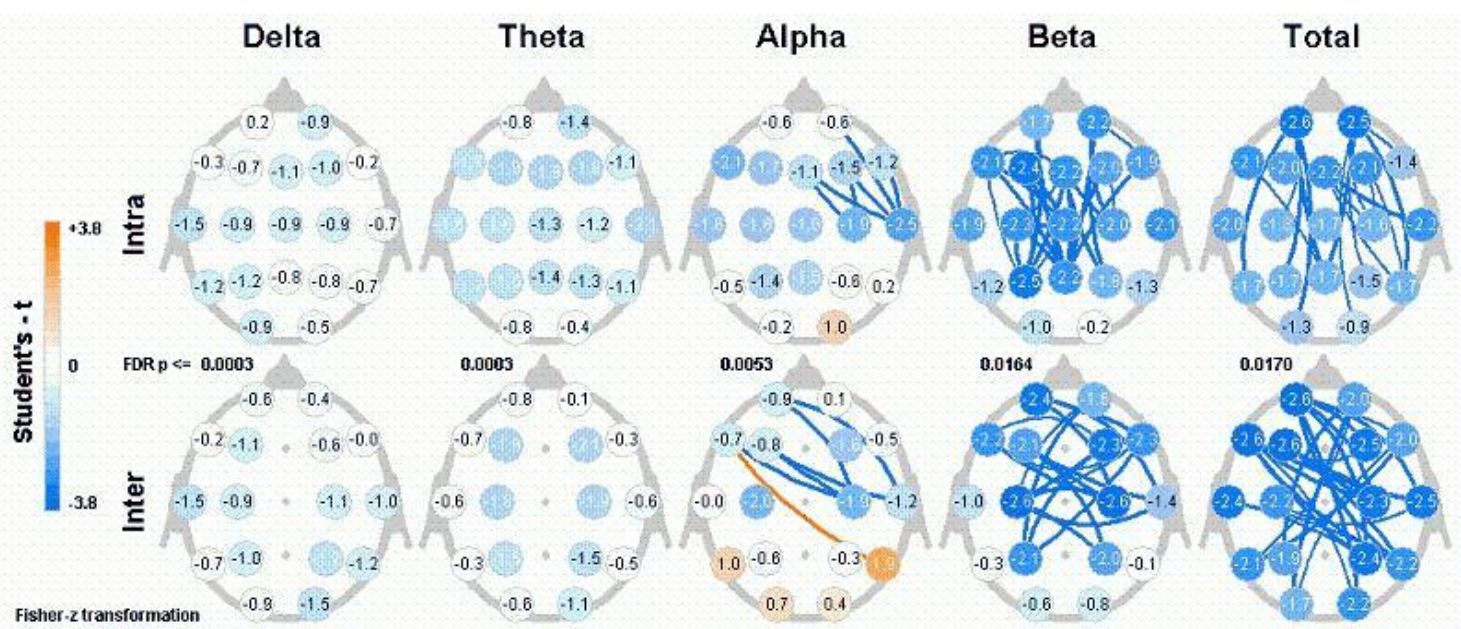

Figure 8. Graphical representation of Compare findings illustrating focal changes in neural connectivity.

eLORETA analysis was also computed for the control group from pre- to post-conditions. Analogous to the experimental group to compare the current density amplitude of the two conditions of the wait-list control, we used the randomization-permutation multiple comparison t-max approach (Congedo et al., 2004). Data-permutation approaches can adaptively account for the correlation structure of the variables, an embedded feature of all electrophysiological measurements (Holmes et al., 1996). Again, we performed one test for each of the 13 frequency band-pass regions (delta, theta, alpha, beta, low frequency, high frequency, alpha1, alpha2, beta1, beta2, beta3, beta4, and beta5). For the whole data set $(2,394 \times 9$ variables), voxel-by-voxel within $t$-tests were computed. This is the $t$-test for paired designs. In this test the mean of two conditions are compared. A threshold of significance (if the global null hypothesis was false) was then computed by the t-max method. For all bands, we tested the hypothesis that the mean LORETA current density amplitude of the two conditions differed by subtracting the values for the pre-condition from the post-condition (Congedo et al., 2004). Further, the results revealed that there were no significant changes from pre- to post-conditions among the participants in the wait-list control group using an alpha level of $p<.10$.

Statistical analyses performed on the experimental group were also implemented for the 12 participants of the control group in order to test for significant changes in coherence. The analysis revealed that from pre- to post-conditions the control group had no significant changes in coherence. Similar to the experimental group analysis, the inclusion of FDR (Benjamini \& Hochberg, 1995) protects this analysis from errors associated with multiple comparisons. Further, the analysis showed that in the control group no electrode location approached a $p<.10$ level, let alone significance. 


\section{Discussion}

The major finding of this study is the evidence that connectivity-guided EEG biofeedback is a treatment capable of causing therapeutic neurophysiological changes in the brains of children with ASD. Significant reductions in coherence between numerous neural regions occurred as a result of this intervention. These findings were further substantiated by the eLORETA analysis, which showed prominent source localized changes in power in crucial regions of the brain for such children. These changes were evident across frontal, temporal, and limbic regions. The results of the current study also support and expand upon the findings found previously (Coben \& Padolsky, 2007). In our earlier study, we achieved an $89 \%$ success rate that was coupled with a $40 \%$ reduction in core ASD symptoms. Moreover, significant improvements were noted for the experimental group on measures of attention, executive, visual perceptual, and language functions. In contrast, our analysis revealed that the control group did not significantly differ from the experimental group at the conclusion of our previous study insomuch that $83 \%$ of the parents of this group reported no change and presently no significant pre- to post-conditions changes were seen. The significant clinical findings previously reported are now bolstered by the neurophysiology changes found presently. This now provides preliminary evidence that connectivity-guided EEG biofeedback is capable of producing neurophysiological changes while concurrently reducing autistic symptoms. Specifically, the findings found previously, coupled with our current findings, provide evidence that seemingly links reductions in hypercoherence and sourcelocalized power with reductions in autistic symptoms.

This has a two-fold importance. First, our analysis showed that the resulting decreases in hypercoherence derived from connectivity-guided EEG biofeedback seemed to conglomerate in and around the frontotemporal region. These were the regions targeted for treatment. This suggests that training over specific regions can have specific, localized effects. Second, this finding provides support to the theory of frontal system involvement in ASD indicated by previous investigations (Courchesne \& Pierce, 2005; Buxhoeveden et al., 2004; Rippon et al., 2007).

The theory of hypercoherence/connectivity, as it relates to autistic symptoms, has been the subject of several investigations. Courchesne and Pierce (2005) reported patterns of overconnectivity within the frontal lobes, as well as under-connectivity between the frontal lobe and other brain regions. Courchesne, Redkay, and Kennedy (2004) previously attributed these findings to abnormal increases in the gray and white matter neurons of the frontal and temporal lobes occurring between the ages of 2 to 4 years old. These abnormal increases in brain matter or early developmental neuroinflammation are thought to cause malfunctions in the brain, particularly in frontal minicolumn microcircuitry (Courchesne \& Pierce, 2005). Neuroinflammation is also thought to explain the finding of enlarged head sizes found in children with ASD (Herbert et al., 2003; Herbert et al., 2004). It is theorized that overconnectivity can result from this neuroinflammation, due to the tendency of neurons that are excited to communicate more readily with other neurons that are close in proximity (Courchesne \& Pierce, 2005). Furthermore, as neural regions expand the neuropil space is reduced, causing an increase in neuron proximity that in turn will increase hyperconnectivity (Buxhoeveden et al., 2004). Likewise, under-connectivity of the frontal cortex to other neural regions is produced due to the hyperconnected brain's inability to form sufficient communications with other areas. Consistent with this, recent findings have indicated that neuroinflammation of white matter impedes the autistic brain's ability to connect or integrate information from other parts of the brain (Herbert, 2005). This inability to communicate efficiently between neural assemblies may result in deficits in domains that require more 
coordination and communication between brain areas, namely language and executive functioning. Further, this phenomenon can readily be described as too many local services competing within themselves, in turn decreasing the quality of long distance communication. These neural anomalies of hyper- and hypoconnectivity in autism have also been prescribed as the consequence of a faulty pruning system. Frith (2003) speculated that the neurophysiological brain deficits associated with autism are the result of a neural pruning system that fails to eliminate faulty connections within the brain during keys stage of development. This pruning system plays a key role in coordinating neural functioning in the healthy individuals. The failure of these inutile connections to be eliminated interferes with normal neural connections both locally and long distance within the brain.

When long-distance frontal neural assembly connections are disrupted, resulting deficits in integration of information from emotional, sensory, language, and automatic systems can occur (Courchesne \& Pierce, 2005). Moreover, disruption of white matter tracts, as described previously, may cause deficit in social cognition associated with neural areas responsible for face and gaze processing, awareness of mental states, and emotional processing (Barnea-Goraly et al., 2004). Further, deficits of frontal cortical networks are thought to lead to executive functioning impairments in areas of personal and social behavior, emotion, and decision-making (Salloway et al., 2001; Courchesne \& Pierce, 2005). Likewise, deficits within the cortical area of the limbic system, most notably the anterior part of the cingulate gyrus, are theorized to result in deficiencies in emotional processing, the control of facial expressions, and the affective dimensions of pain (Williams et al., 2005). Insufficiencies within the temporal lobe, particularly the amygdala, have been correlated with autistic impairment related to social functioning and behavior (Baron-Cohen et al., 2000). Finally, by therapeutically reducing hypercoherence within these neural areas, as was achieved in this study, it is thought that the previously mentioned deficits and pathophysiology can be reduced and that positive behavioral changes can be gained.

Our current study contributes to the aforementioned research by presenting evidence that shows that reducing hypercoherence may play an integral role in the improved treatment outcomes that result from connectivity-guided EEG biofeedback. The evidence presented linking reductions in frontotemporal hypercoherence, as a means to produce therapeutic gains in autistics, is further confirmation of the critical roles these brain regions play in the symptoms of autistic disorders.

This is the first study to present evidence of a treatment of ASD that is capable of therapeutically changing the neurophysiological dysfunction that is at the heart of autistic symptoms. The decreases in neural coherence that were achieved in the present study included frontal, temporal, and underlying limbic structures. As stated previously, these areas have been confirmed to be associated with autistic impairment (Salloway et al., 2001; Courchesne \& Pierce, 2005; Williams et al., 2005; Baron-Cohen et al., 2000). As such, the positive gains achieved by therapeutically impacting these neural areas confirms their involvement in ASD, as well as provides an insight into the overall neurophysiological mechanisms responsible for the efficacy of this therapy.

The significant neurophysiological changes reviewed can be ascribed to the treatment with little risk of error due to the implementation of FDR indices (Benjamini \& Hochberg, 1995) and randomization-multiple permutation analyses (Congedo et al., 2004). Moreover, these changes can be confidently regarded as ameliorative in nature due to subjects' reports of positive therapeutic gains and reductions in autistics symptoms at the completion of 20 sessions of connectivity-guided EEG biofeedback. These results are further strengthened by 
our analysis, which revealed that the wait-list control group reported no significant changes from pre- to post-conditions.

Our analysis showed that there were positive changes in source-localized power (eLORETA) within lower frequency bands; while findings also indicated that changes in coherence were seen predominately in the higher frequencies of alpha and beta band. The reason for this discrepancy is not yet clearly understood. Further, it would take increasingly more investigation to fully understand the implications of this difference.

Recently, Pineda et al. (2007) investigated behavioral changes as a result of neurofeedback in children with autism. The results showed that indeed positive behavioral changes were seen within the Speech/Language, Sociability, and Health/Physical behavior subscales of the ATEC as well as the Total score. However, when comparing this data to the current investigation, it was revealed that the intervention implemented presently produced about a three times greater rate of efficacy. Moreover, our current study saw no degradation or worsening of symptoms as assessed by any subscales of the ATEC. Conversely, Pineda et al. (2007) presented evidence of negative changes in behavior in terms of the

Sensory/Cognitive Awareness subscale of the ATEC. Continuing, Pineda et al. (2007) chose to train the Mu rhythm mainly on the right hemisphere of the brain focusing on electrode site C4 as well as areas chosen based on EMG activity. This technique produced a significant decrease in coherence for only one neural pair (C3-C4; Study 1) and three neural pairs (T3 -T4; C3-C4; F3-F4) for study two (Pineda et al., 2007). We, on the other hand, based the course of therapy on each individual's QEEG analysis which revealed specific areas of maximinal hypercoherence and we postulate that this served as an underlying reason why our investigation was able to significantly decrease coherence between far more neural pairs (Refer to Fig. 5 through 7). We would prescribe our increased success and lack of negative effects to our use of personalized QEEG analysis to plan and carry out neurofeedback, resulting in far greater reductions. Similarly, Coben and Myers (2008) have recently compared data from their connectivity-guided EEG biofeedback study to Jarusiewicz's (2002) earlier symptom-based neurofeedback investigation. The results of this analysis indicated that connectivity-guided EEG biofeedback accomplished, on average, a full standard deviation greater improvement as compared to symptom-based neurofeedback, while still preventing any unwanted effects. The implication of the aforementioned research comparisons would suggest that personalization of EEG protocols among subjects plays a positive role in the efficacy of our treatment.

Others have recently hypothesized that the positive effects of neurofeedback for individuals with autism might be due to non-specific factors (Heinrich, Gevensleben, \& Strehl, 2007; Kouijzer, van Schie, Gerrits, Buitelaar, \& de Moor, 2013). For example, Kouijzer et al. (2013) showed no difference in effect between EEG biofeedback and skin conductance biofeedback, but both were better in reducing autistic symptoms as compared to a nontreatment control group. They concluded that the beneficial effects might be due to treatment expectancy, implicit training of attention and/or intensive one-to-one contact with a therapist. However, their training was only at midline locations and did not involve connectivity or coherence training. Our current findings indicate improvements in clinical functioning associated with treatment-related neurophysiological changes in brain functioning that did not occur in the wait-list comparison group. This indicates a strong likelihood that there were specific effects from the training and that the mechanism of action is the alteration of coherence in a therapeutic direction. Interestingly, different types of neurofeedback trainings may have different impacts. 
In terms of the limitations of our study, the participants consisted of a selected pool of subjects. When subjects or, in this case, parents of subjects, select their preferred treatment, there is a risk of selection bias that may interact with the treatment effect. Therefore, randomized assignment of experimental and control groups would be needed to test for any interactions between the treatment effect and the subject selection. Also, efforts could be made to include more subjects with increased severity of symptoms as well as more homogeneity in terms of age. This would help better assess whether the severity of autistic symptoms moderates the efficacy of the treatment. Additionally, to more precisely measure the effects of our treatment, a double-blind study design, in which subject assignment would be unknown by both the subjects and experimenters, would be recommended. Furthermore, the addition of an alternative treatment or a placebo-controlled (i.e., sham neurofeedback) comparison group could help better assess the efficacy of connectivity-guided EEG biofeedback and help demonstrate that our results were not likely due to chance or an uncontrolled variable. Periodic future follow-up assessments would also be beneficial in determining the efficacy of our intervention over an extended period of time. This would help better demonstrate the continuing efficacy over time of connectivity-guided EEG biofeedback. We would also recommend future studies more precisely analyze the relationship between connectivity/coherence and autism. This investigation provided evidence linking these two concepts to each other and to autistic impairments; however, further research would need to be conducted to enduringly validate this claim. Finally, the addition of alternative imaging techniques (i.e., MRI or DTI), used in tandem with EEG analysis, would help better validate the neurophysiological changes found presently.

\section{References}

American Psychiatric Association. (1994). Diagnostic and statistical manual of mental disorders (4th ed.). Washington, DC: Author.

Baron-Cohen, S. (2004). The cognitive neuroscience of autism. Journal of Neurology, Neurosurgery \& Psychiatry, 75, 945-948. http://dx.doi.org/10.1136/jnnp.2003.018713

Baron-Cohen, S., Ring, H. A., Bullmore, E. T., Wheelwright, S., Ashwin, C., \& Williams, S. C. (2000). The amygdale theory of autism. Neuroscience and Biobehavioural Reviews, 24, 355-364. http://dx.doi.org/10.1016/S0149-7634(00)00011-7

Barnea-Goraly, N., Kwon, H., Menon, V., Eliez, S., Lotspeich, L., \& Reiss, A.L. (2004). White matter structure in autism: Preliminary evidence from diffusion tensor imaging. Biological Psychiatry, 55, 323-326. http://dx.doi.org/10.1016/j.biopsych.2003.10.022

Belmonte, M. K., Allen, G., Beckel-Mitchener, A., Boulanger, L. M., Carper, R. A., \& Webb, S. J. (2004). Autism and abnormal development of brain connectivity. The Journal of Neuroscience, 24(42), 9228-9231. http://dx.doi.org/10.1523/JNEUROSCI.3340-04.2004

Benjamini, Y. \& Hochberg, Y. (1995). Controlling the false discovery date: A practical and powerful approach to multiple testing. Journal of the Royal Statistical Society, 57, 289-300. 


\section{NeuroRegulation}

Bendat, J. S. \& Piersol, A. G. (1980). Engineering applications of correlation and spectral analysis. New York: John Wiley and Sons.

Brodmann, K. (2005). Brodmann's: Localisation in the cerebral cortex. (L.J. Garey, Trans.). New York: Springer. (Original work published 1909).

Buxhoeveden, D., Semendeferi, K., Schenker, N., \& Courchesne, E. (2004). Decreased cell column spacing in autism. Social Neuroscience, 582, 6.

Cantor, D. S., Thatcher, R. W., Hrybyk, M., \& Kaye, H. (1986). Computerized EEG analyses of Autistic children. Journal of Autism and Developmental Disorders, 16, 169-187.

Center for Disease Control and Prevention (2014). Prevalence of Autism Spectrum Disorder Among Children Aged 8 Years - Autism and Developmental Disabilities Monitoring Network, 11 Sites, United States, 2010. Morbidity and Mortality Weekly Report, 63(SS02), 1-21.

http://www.cdc.gov/mmwr/preview/mmwrhtml/ss6302a1.htm?s cid=ss6302a1 w

Coben, R. (2007). Connectivity-guided neurofeedback for autistic spectrum disorder. Biofeedback, 35(4), 131-135.

Coben, R., Chabot, R. J., \& Hirschberg, L. (2013). EEG analyses in the assessment of autistic disorders. In Cassanova, M. F., Ayman, S. E., Suri, J. (Eds.), Imaging the Brain in Autism (349-369). Springer Science + Business Media New York.

Coben, R. Clarke, A. R., Hudspeth, W., \& Barry, R. J. (2008). EEG power and coherence in autistic spectrum disorder. Clinical Neurophysiology, 119, 1002 http://dx.doi.org/10.1016/i.clinph.2008.01.013

Coben, R., \& Myers, T. E. (2008). Connectivity theory of autism: Use of connectivity measures in assessing and treating autistic disorders. Journal of Neurotherapy, 12, 161-179. http://dx.doi.org/10.1080/10874200802398824

Coben, R., \& Padolsky, I. (2007). Assessment-guided neurofeedback for autistic spectrum disorder. Journal of Neurotherapy, 11, 5-23. http://dx.doi.org/10.1300/J184v11n01 02

Collins D. L., Neelin P., Peters T. M., \& Evans A. C. (1994). Automatic 3D intersubject registration of MR volumetric data in standardized Talairach space. Journal of Computer Assisted Tomography, 18, 192-205.

Congedo, M., Finos, L., \& Turkheimer, F. (2004). A multiple hypothesis test procedure based on the sum of test-statistics, 10th Annual Meeting of the Organization for Human Brain Mapping, June 13-17, Budapest, Hungary. (Abstract) on CD.

Courchesne, E., Recay, E., \& Kennedy, D. P. (2004). The autistic brain: Birth through adulthood. Current Opinion in Neurology, 17(4), 489-496. http://dx.doi.org/10.1097/01.wco.0000137542.14610.b4 


\section{NeuroRegulation}

Courchesne, E., \& Pierce, K. (2005). Why the frontal cortex in autism might be talking only to itself: Local over-connectivity but long-distance disconnection. Current Opinion in Neurobiology, 15, 225-230. http://dx.doi.org/10.1016/j.conb.2005.03.001

Deymed Diagnostic (2004). TruScan 32 Specifications. Retrieved June 16, 2008 from http://www.deymed.com/truscan32.asp

Egner, T. \& Sterman, M. B. (2006). Neurofeedback treatment of epilepsy: From basic rationale to practical application. Expert Review of Neurotherapeutics, 6(2), 247-257. http://dx.doi.org/10.1586/14737175.6.2.247

Evans, J. R., \& Park, N. S. (1996). Quantitative EEG abnormalities in a sample of dyslexic persons. Journal of Neurotherapy, 2(1), 1-5.

Frei, E., Gamma, A., Pascual-Marqui, R. D., Lehmann, D., Hell, D., \& Vollenwider, F. X. (2001). Localization of MDMA-induced brain activity in healthy volunteers using low resolution brain electromagnetic tomography (LORETA). Human Brain Mapping, 14, 152-165. http://dx.doi.org/10.1002/hbm.1049

Filipek, P. A., Accardo, P. J., Ashwal, S., Baranek, G. T., Cook, E. H., Dawson, G., ..., Volkmar, F. R. (1999). Practice parameter: Screening and diagnosis of autism. Journal of Autism and Developmental Disorders, 29(6), 439-484.

Frith, C. D. (2003) What do imaging studies tell us about the neural basis of autism? In Autism: neural basis and treatment possibilities (ed. M. Rutter), Novartis Foundation. Chichester, UK: Wiley.

Ganz, M. (2006). The costs of autism. In Moldin, S. \& Rubenstein, J. L. (Ed.), Understanding autism: from basic neuroscience to treatment (pp. 476-498). New York, NY: CRC Press.

Gilliam, J. E. (1995). Gilliam Autism Rating Scale Examiner's Manual. Austin, Texas: Pro-Ed.

Gilliam, J. E. (2001). Gilliam Asperger's Disorder Scale Examiner's Manual. Austin, Texas: Pro-Ed.

Gioia, G. A., Isquith, P. K., Guy, S. C., \& Kenworthy, L. (2000). Behavior Rating Inventory of Executive Function. Lutz, FL:.Psychological Assessment Resources, Inc.

Grass SafeLead (2006). Genuine grass precious metal recording electrodes: SafeLead. Retrieved June 17, 2008 from http://www.grasstechnologies.com/

Hammond, D. C. (2007). What is neurofeedback? Journal of Neurotherapy, 10(4), 25-36. http://dx.doi.org/10.1300/J184v10n04 04

Heinrich, H, Gevensleben, H. \& Strehl, U. (2007). Annotation: Neurofeedback - train your brain to train behavior. Journal of Clinical Psychology and Psychiatry, 48(1), 3-16. http://dx.doi.org/10.1111/j.1469-7610.2006.01665.x 
Herbert, M.R., Ziegler, D.A., Makris, N., Bakardjiev, A., Hodgson, J., Adrien, K.T.,,... Caviness, V.S. (2003). Larger brain and white matter volumes in children with developmental language disorder. Developmental Science, 6(4), F11-F22. http://dx.doi.org/10.1111/1467-7687.00291

Herbert, M. R., Ziegler, D. A., Makris, N., Filipek, P. A., Kemper, T. L., Normandin, J. J., ... Caviness, V. S. (2004). Localization of white matter volume increase in autism and developmental language disorder. Annals of Neurology, 55, 530-540. http://dx.doi.org/10.1002/ana.20032

Herbert, M.R. (2005). Large brains in autism: The challenge of pervasive abnormality The Neuroscientist, 11(5), 417-440. http://dx.doi.org/10.1177/0091270005278866

Holmes, A. P., Blair, R. C., Watson, J. D. G., \& Ford, I. (1996). Nonparametric analysis of statistic images from functional mapping experiments. Journal of Cerebral Blood Flow \& Metabolism, 16, 7-22.

Hudspeth, W. J. (1999). NeuroRep QEEG analysis and report software (Version 4.0) [Computer Software]. Los Osos, CA: Grey Matter Inc.

Jarusiewicz, B. (2002). Efficacy of neurofeedback for children in the autistic spectrum. A pilot study. Journal of Neurotherapy, 6(4), 39-49.

http://dx.doi.org/10.1300/J184v06n04 05

John, E. R., Prichep, L. S, \& Easton, P. (1987). Normative data banks and neurometrics: Basic concepts, method and results of norm constructions in method of analysis of brain electrical and magnetic signals. In: Gevins, A. S. \& Remond A., (Ed.)., EEG Handbook (revised series. Vol.1), New York: Elsevier Science Publishers.

John, E. R., Prichep, L., Fridman, J., \& Easton, P. (1988). Neurometrics: Computer-assisted differential diagnosis of brain dysfunction. Science, 239, 162-169.

Koechlin, E. \& Hyafil, A. (2007). Anterior prefrontal function and the limits of human decisionmaking. Science, 311, 594-598. http://dx.doi.org/10.1126/science.1142995

Kouijzer, M. E., van Schie, H. T., Gerrits, B. J. L., Buitelaar, J. K., \& de Moor, J. M. H. (2013). Is EEG-Biofeedback an effective treatment in autism spectrum disorders? A randomized controlled trial. Appl Psychophysiol Biofeedback, 38, 17-28. http://dx.doi.org/:10.1007/s10484-012-9204-3

Lachar, D., \& Gruber, C. P. (2001). The personality inventory for children, second edition. Los Angeles, CA: Western Psychological Services.

Leins, U., Goth, G., Hinterberger, T., Klinger, C., Rumpf, N., \& Strehl, U. (2007). Neurofeedback for children with ADHD: A comparison of SCP and Theta/Beta protocols. Applied Psychophysiology and Biofeedback, 32, 73-88. http://dx.doi.org/10.1007/s10484-007-9031-0 
Lubar, J. F., Swartwood, M. O., Swartwood, J. N., \& O'Donnell, P. H. (2005). Evaluation of the effectiveness of EEG neurofeedback training for ADHD in a clinical setting as measured by changes in T.O.V.A. scores, behavioral ratings, and WISC-R performance. Applied Psychophysiology and Biofeedback, 20, 83-99. http://dx.doi.org/10.1007/BF01712768

Lubar, J. F., Congedo M., \& Askew, J. H. (2003). Low-resolution electromagnetic tomography (loreta) of cerebral activity in chronic depressive disorder. International Journal of Psychophysiology, 49, 175-185. http://dx.doi.org/10.1016/S0167-8760(03)00115-6

Miller, C.J., Genovese, C., Nichol, R.C., Wasserman, L., Connolly, A., Reichart, D., Hopkins, A., Schneider, J. \& Moore, A. (2001). Controlling the false discovery rate in astrophysical data analysis. The Astronomical Journal, 122, 3492-3505.

Mizuno, A., Villalobos, M. E., Davies, M. M., Dahl, B. C., \& Muller, R. A. (2006). Partially enhanced thalamocortical functional connectivity in autism. Brain Research, 1104, 160-174. http://dx.doi.org/10.1016/j.brainres.2006.05.064

Monastra, V. J., Monastra, D. M., \& George, S. (2002). The effects of stimulant therapy, EEG biofeedback, and parenting style on the primary symptoms of attentiondeficit/hyperactivity disorder. Applied Psychophysiology and Biofeedback, 27, 231249.

Murias, M., Webb, S. J., Greenson, J., \& Dawson, G. (2007). Resting state cortical connectivity reflected in EEG coherence in individuals with autism. Biological Psychiatry, 62, 270-273. http://dx.doi.org/10.1016/j.biopsych.2006.11.012

NeuroCybernetics Inc. (2006). Specific EEGer technical parameters. Canoga Park, CA: NeuroCybernetics, Inc.

NxLink (2001). Neurometric analysis system. Richland, WA: Nxlink Ltd.

Pascual-Marqui, R. D. (1999). Review of methods for solving the EEG inverse problem. International Journal of Bioelectromagnetism, 1(1), 75-86.

Pascual-Marqui, R. D. (2007). Discrete, 3D distributed linear imaging methods of electric neuronal activity. Part I: exact, zero localization. Retrieved October 16, 2008 from http://www.uzh.ch/keyinst/eLORETA/eLorOerrLikeltOrNot.pdf

Pascual-Marqui, R. D., Esslen, M., Kochi, K., \& Lehmann, D. (2002). Functional imaging with low resolution brain electromagnetic tomography (LORETA): A review. Methods \& Findings in Experimental \& Clinical Pharmacology, 24C, 91-95.

Pascual-Marqui, R. D., Michel, C. M., \& Lehmann, D. (1994). Low resolution electromagnetic tomography: A new method for localizing electrical activity in the brain. International Journal of Psychophysiology, 18, 49-65.1.

Rimland, B., \& Edelson, S. M. (2000). Autism treatment evolution checklist (ATEC). Retrieved October 13, 2008 from https://www.autismeval.com/ari-atec/report1.html 


\section{NeuroRegulation}

Rippon, G., Brock, J., Brown, C., \& Boucher, J. (2007). Disordered connectivity in the autistic brain: Challenges for the "new psychophysiology". International Journal of Psychophysiology, 63, 164-172. http://dx.doi.org/10.1016/.i.jpsycho.2006.03.012

Safran, S.P. (2008). Why youngsters with autistic spectrum disorders remain underrepresented in special education. Remedial and Special Education, 29(2), 9095. http://dx.doi.org/10.1177/0741932507311637

Salloway, S. P., Malloy, P. F., \& Duffy, J. D. (2001). The frontal lobes and neuropsychiatric illness. Washington D.C.: American Psychiatric Publishing, Inc.

Sherlin, L., Budzynski, T., Budzynski, H. K., Congedo, M., Fischer, M. E., \& Buchwalk, D. (2007). Low-resolution electromagnetic brain tomography (LORETA) of monozygotic twins discordant for chronic fatigue syndrome. Neurolmage, 34(4), 1438-1442. http://dx.doi.org/10.1016/j.neuroimage.2006.11.007

Talairach J, \& Tournoux, P. (1988). Co-planar stereotaxic atlas of the human brain: Threedimensional proportional system. Georg Thieme: Stuttgart.

Thatcher, R. W., Walker, R. A., Biver, C. J., North, D. N., \& Curtin, R. (2003). Quantitative EEG normative databases: Validation and clinical correlation. In Lubar, J. F. (Ed.) Quantitative electroencephalographic analysis (QEEG) databases for neurotherapy: Descriptions, validation, and applications (pp.87-121). New York: Haworth Press.

Thatcher, R. W., Krause, J. \& Hrybyk (1986). Cortico-cortical associations and EEG coherence: a two-comportmental model. Electroencephalography and Clinical Neurophysiology, 64, 123-143.

Towle, V. L., Bolaños, J., Suarez, D., Tan, K., Grzeszczuk, R., Levin, D. N., Cakmur, R., Frank, S. A., \& Spire, J. (1993). The spatial location of EEG electrodes: Locating the best fitting sphere relative to cortical anatomy. Electroencephalography and Clinical Neuroscience, 86:1-6.

Walker, J. E., Kozlowski, G. P., \& Lawson, R. (2007). A modular activation/coherence approach to evaluating clinical/QEEG correlations and for guiding neurofeedback training: Modular insufficiencies, modular excesses, disconnections, and hyperconnections. Journal of Neurotherapy, 11, 25-44. http://dx.doi.org/10.1300/J184v11n01 03

Williams, S. M., White, L. E., \& Mace, A. C. (2005). SylviusVG: Visual glossary of human neuroanatomy. Sunderland, MA: Puramis Studios, Inc.

Yeargin-Allsopp, M., Rice, C., Karapurkar, T., Doernberg, N., Boyle, C. \& Murphy, C. (2003). Prevalence of autism in a US metropolitan area. Journal of American Medical Association, 289(1), 49-55. http://dx.doi.org/10.1001/jama.289.1.49 